Transportation Research Forum

Characteristics of Vehicle Platoons at Isolated Intersections

Author(s): Yi Jiang and Shuo Li

Source: Journal of the Transportation Research Forum, Vol. 44, No. 1 (Spring 2005), pp. 5-25

Published by: Transportation Research Forum

Stable URL: http://www.trforum.org/journal

The Transportation Research Forum, founded in 1958, is an independent, nonprofit organization of transportation professionals who conduct, use, and benefit from research. Its purpose is to provide an impartial meeting ground for carriers, shippers, government officials, consultants, university researchers, suppliers, and others seeking exchange of information and ideas related to both passenger and freight transportation. More information on the Transportation Research Forum can be found on the Web at www.trforum.org. 


\section{CHARACTERISTICS OF VEHICLE PLATOONS AT ISOLATED INTERSECTIONS}

It was observed that about $70 \%$ of vehicles travel in platoons on Indiana highway corridors in the vicinity of urban areas. Many vehicle platoons were halted at signalized intersections to give the right of way to vehicles on the intersecting minor road, even though there were only one or two vehicles on the minor road. This phenomenon suggested that the total traffic delay at an intersection might be reduced if the approaching vehicle platoons on the major road were allowed to pass through the intersection at the expense of a reasonably prolonged delay of the few vehicles on the minor road. Consequently, a study was conducted to investigate vehicle platoon characteristics and to develop control logic for timing actuated traffic signals in terms of the presence of vehicle platoons. This paper presents some findings of the study on traffic flows in terms of vehicle platoons. Included in this paper are the selection of the fundamental platoon variables and determination of the critical headway value for platoon identification, the appropriate platoon detector placement, and the mathematical distributions of the platoon variables.

\section{by Yi Jiang and Shuo Li}

There are three main types of intersection signal controls: pre-timed signal control, semiactuated signal control, and fully actuated signal control (McShane, Roess, and Prassas 1998). In terms of signal timings, there are two types of intersections, i.e., coordinated intersections and isolated intersections. Coordinated intersections consist of two or more adjacent intersections that are timed so that when a queue of vehicles is released by receiving the right of way at an intersection, these vehicles will also have the right of way at the adjacent intersections. The coordinated signal timing is often used in urban areas so that the coordination will reduce the delay experienced by the vehicles on the arterial routes. An isolated intersection is one in which the signal time is not coordinated with that of any other intersection and therefore operates independently (Garber and Hoel 1996). The purpose of this study was to analyze the characteristics of vehicle platoons at isolated intersections.

The current methods of signal timings of signal controls are all based on traffic volumes in terms of individual vehicles. However, it was observed that about $70 \%$ of vehicles travel in platoons on Indiana highway corridors in the vicinity of urban areas. Many vehicle platoons were halted at signalized intersections to give the right of way to vehicles on the intersecting minor road, even though there were only one or two vehicles on the minor road. Thus, a great portion of the traffic delay at a certain intersection was caused by the stopped vehicle platoons. It also suggested that the total traffic delay at an intersection might be reduced if the approaching vehicle platoons on the major road were allowed to pass through the intersection at the expense of a reasonably prolonged delay of the few vehicles on the minor road.

The results presented in this paper provide a basis for deriving platoon-based traffic control algorithms, for formulizing platoon-generating patterns, and for developing platoon simulation programs. More importantly, they provide a foundation to analyze traffic flows in terms of vehicle platoons rather than individual vehicles. It is believed that a new method for signal design and timing based on vehicle platoons would lead to a considerable reduction of traffic delays at isolated intersections because of the observation that the majority of vehicles travel in groups on rural and semi-rural highways with isolated intersections. This would also improve economic efficiency and minimize the opportunity cost of time because travel time of the vehicle 
population would be reduced. In addition, it would also improve traffic safety and reduce vehicle-produced air pollution because of more stable vehicle speed.

A vehicle platoon is defined as a group of vehicles traveling together according to the Highway Capacity Manual (HCM) (TRB 2000). HCM uses a platoon ratio, $R_{p}$, to take into account the presence of platoons. $R_{p}$ is calculated as $R_{p}=P(C / g)$, where $P$ is the proportion of all vehicles in movement arriving during the green signal phase, $\mathrm{C}$ is the traffic signal's cycle time, and $\mathrm{g}$ is the effective green time for the movement. $\mathrm{R}_{\mathrm{p}}$ in $\mathrm{HCM}$ is utilized as a measure of traffic progression quality at intersections. A favorable progression quality would be that a large portion of vehicles arrive at the intersection during the green signal phase. HCM specifies that if $R_{p} \leq 0.50$, the progression quality is very poor, and if $\mathrm{R}_{\mathrm{p}}>2.00$, it is exceptional. However, $\mathrm{R}_{\mathrm{p}}$ values are not considered in signal design and timing. Furthermore, $\mathrm{R}_{\mathrm{p}}$, as discussed in $\mathrm{HCM}$, is so simplified that the essential characteristics of vehicle platoons are omitted. As observed in the field, the portion of vehicles traveling in groups changes from time to time, and is not a constant. An arbitrary value of platoon ratio cannot represent the real platoon characteristics.

The literature shows that some researchers started to pay attention to vehicle platoons and developed methods for platoon recognitions. A study by the Texas Transportation Institute (Chaudhary 2003) used a platoon identification system to optimize traffic flow. Gaur and Mirchandani (2001) developed an algorithm to identify vehicle platoons from traffic-density data on highway network links.

To incorporate vehicle platoons into signal design and timing, it is desirable to identify major variables of vehicle platoons and represent their characteristics mathematically. Consequently, a platoon-based signal control algorithm can be developed. Therefore, this study was conducted to investigate vehicle platoon characteristics and to develop control logic for timing actuated traffic signals at isolated intersections in terms of the presence of vehicle platoons. This paper presents some findings of the study on traffic flows in terms of vehicle platoons. Included in this paper are the selection of the fundamental platoon variables, determination of the critical headway value for platoon identification, the appropriate platoon detector placement, and the mathematical distributions of the platoon variables.

\section{MEASUREMENTS OF PLATOON- BASED TRFFIC DATA}

\section{Selection and Definition of Platoon Variables}

For the purpose of this study, the intersections for platoon data collection were specified as isolated intersections of a major road and a minor road with significant platoon presence on the major road. Tube traffic counters were used to obtain vehicle platoon data. As traffic variables include traffic flow rate, speed and density in the conventional traffic signal timing, four fundamental variables were selected in this study to analyze the characteristics of platoonbased traffic flows. Figure 1 shows a graphical illustration of the four vehicle platoon variables: platoon size, platoon headway, platoon speed, and inter-arrival between consecutive platoons.

As shown in the figure, $\mathrm{D}$ is the distance between the stop line and the platoon detector, the platoon size $\mathrm{n}$ is the number of vehicles in a platoon, the platoon headway $h_{i}$ is the headway

Figure 1: Illustration of Platoon Variables

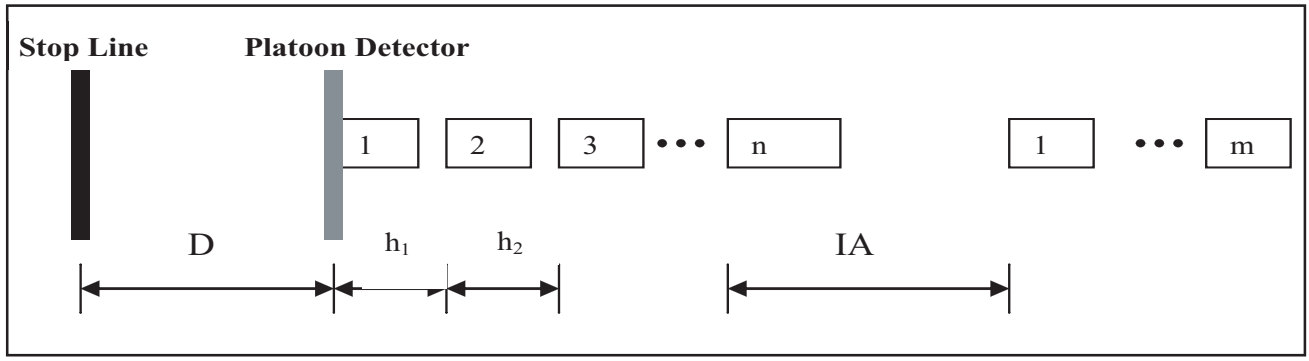


time between vehicle $\mathrm{i}$ and vehicle $\mathrm{i}+1$ within a platoon, the platoon speed $\mathrm{V}$ is the average speed of the vehicles in a platoon, the inter-arrival between consecutive platoons IA is the headway between the last vehicle of the front platoon and the first vehicle of the following platoon. Headway value is essential for determining whether a vehicle belongs to a platoon. That is, if the headway $h_{i}$ is "small," then vehicle $i$ and vehicle $i+1$ belong to the same vehicle platoon. Otherwise, they are not in the same vehicle platoon. To quantify this "small" headway, a pre-determined headway value, or the critical headway as defined by Athol (1965), should be selected. As discussed in the following section, the value of critical headway was determined based on the traffic data collected at selected intersections along Indiana highway corridors.

\section{Determination of Critical Headway}

It is important to select a proper value of the critical headway because a small change in the critical headway will generate tremendous changes in the resultant platoon characteristics. May (1965) investigated individual headway distributions and concluded that vehicle headways were rarely less than 0.5 seconds or more than 10 seconds at different traffic volumes. Athol (1965) investigated the effects of critical headways of 1.2, 1.5, 2.1 and 2.7 seconds on platoon behavior and selected a critical headway of 2.1 seconds corresponding to a traffic volume of 1500 vehicles per hour per lane (vphpl).

To identify an appropriate critical headway, approximately 30,000 headway measurements at the selected Indiana intersections were examined with respect to critical headway values of 1.5 , 2.0, 2.5, 3.0 and 3.5 seconds. Figure 2 shows the frequencies of the resultant vehicle platoon sizes (platoons with two or more vehicles). It shows that vehicle platoons were dominated by the two-vehicle platoons. The two-vehicle platoons increase as the critical headway decreases. When the critical headway is 3.5 seconds, two-vehicle platoons account for about $45 \%$ of the total number of platoons with two or more vehicles. This percentage increases to $74 \%$ when the critical headway drops to 1.5 seconds.

As the critical headway increases, more vehicles will be included in platoons. Use of a large critical headway will result in platoon sizes that are too large and variances of platoon variables that are too large. In addition, detecting large vehicle platoons requires a large detection area, leading to a significant rise in the costs of detector installation and maintenance. The result of a very large critical headway is that every vehicle belongs to a vehicle platoon. On the other hand, use of a small critical headway will result in small platoon sizes and insufficient platoon information. The result of a very small critical headway is that no vehicle belongs to a vehicle platoon, or no platoons can be identified.

Figure 2: Distributions of Platoon Size by Different Critical Headways

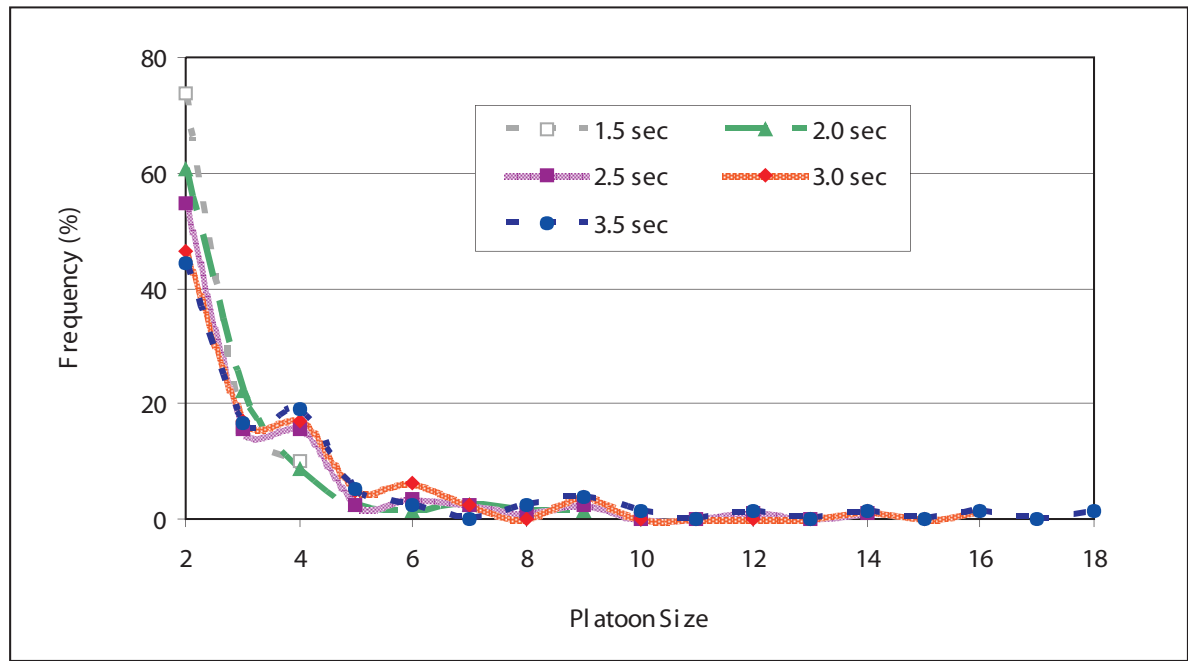


Therefore, use of either a too large or a too small critical headway will not serve the purpose of an effective traffic control in terms of vehicle platoons.

To choose an appropriate value of the critical headway, the variance of platoon size should be kept at a reasonable level so that traffic data contains sufficient and accurate platoon information for signal timing. Therefore, the relationship between the platoon size and the coefficient of variation of the platoon size was examined to determine a proper value of critical headway. The coefficient of variation (COV) of platoon size is defined as the ratio of the standard deviation of platoon size to the average platoon size. Figure 3 shows the proportions of platooned vehicles and COV values corresponding to different critical headways. It is observed that both proportion and $\mathrm{COV}$ values increase as the critical headway increases. As illustrated in Figure 3, the two curves join and inflect at the 2.5-second critical headway. Both curves become flat beyond the inflection point at the 2.5second critical headway. This implies that use of the 2.5-second critical headway would make the proportion of vehicles in platoons and the $\mathrm{COV}$ relatively stable. Consequently, 2.5 seconds is utilized as the critical headway for platoon determination in the data analysis. This critical headway seems to be practically reasonable as it is right in the middle of the commonly assumed saturated headway ( 2.0 seconds) and the desired allowable gap (3.0 seconds) (May 1990).

\section{Determination of Platoon Detector Location}

In conventional signal timing, vehicle detectors should be placed at an optimal distance of $\mathrm{L}$ upstream of the stop line. These detectors are used to determine arrivals of vehicles at the intersection. To acquire platoon information, an additional detector is required on each main approach. Figure 4 illustrates the placement of both the conventional vehicle detector and the additional platoon detector. In the figure, $\mathrm{L}_{\mathrm{p}}$ is the distance for the platoon detector, $\mathrm{L}$ is the distance for the conventional detector, and $\Delta \mathrm{L}$ is the distance between the two detectors.

The location of a vehicle detector is a function of vehicles' approach speed. The Institute of Transportation Engineers (ITE) (ITE 1976) recommended the vehicle detector distance for large-area individual vehicle detection with high-speed approaches as listed on page 9 , with the values converted to the equivalent in metric units from the original U.S. traditional units.

\section{Figure 3: Proportion of Platooned Vehicles and COV at Various Critical Headways}

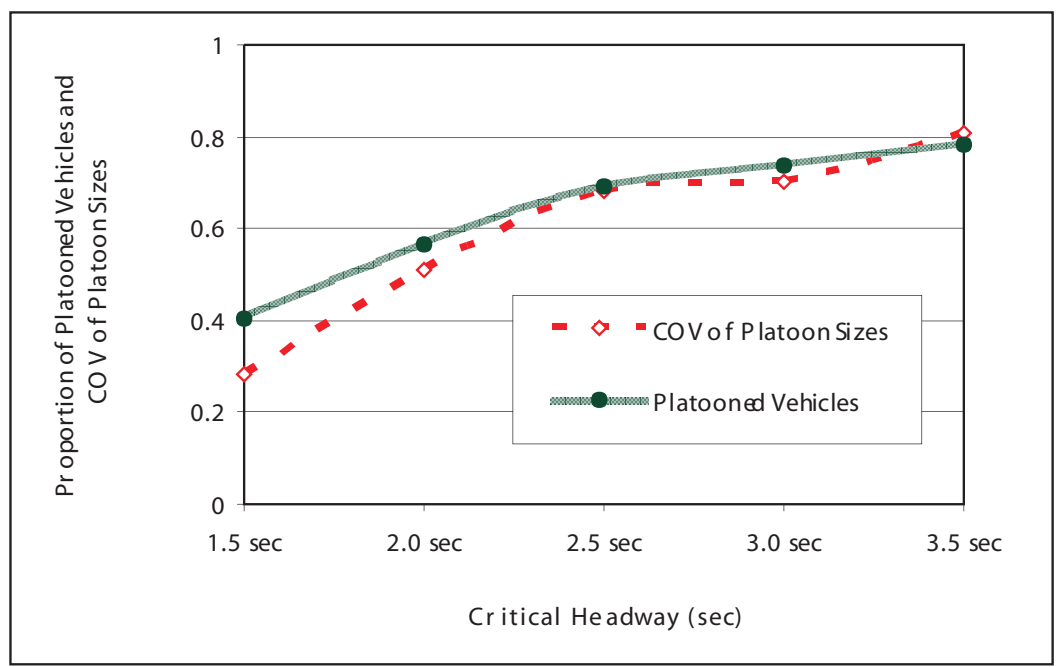


Figure 4: Platoon Detector Placement

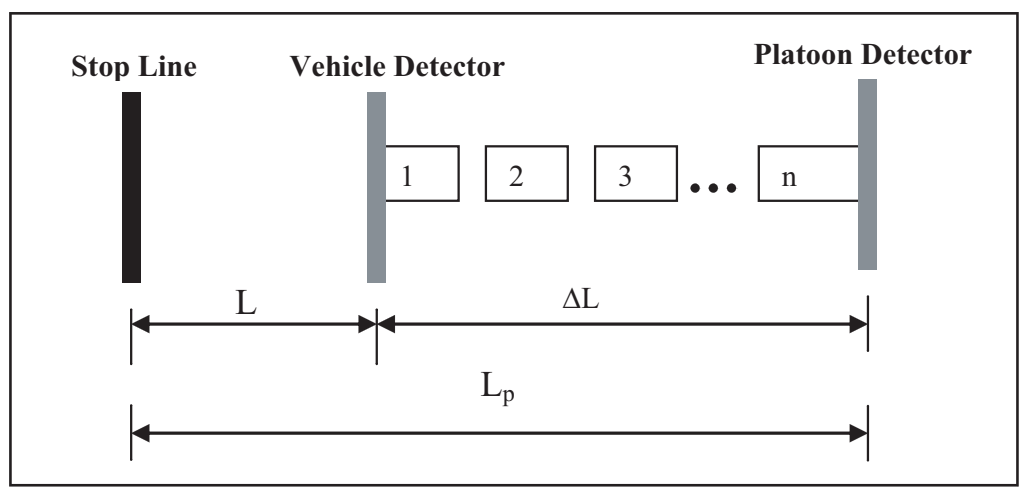

Approach Speed (kilometers per hour)

48
64
72
80
97

Vehicle Detector Distance (meters)

$$
\begin{aligned}
& 53 \\
& 76 \\
& 91 \\
& 107 \\
& 137
\end{aligned}
$$

ITE (1974) also provided the following equation to determine the vehicle detector distance for small-area detection of individual vehicles with low-speed approaches (less than $40 \mathrm{~km} / \mathrm{h}$ ).

$$
\text { (1) } \mathrm{L}=0.28 \mathrm{~V}(3-\mathrm{UE})-5.5
$$

where $\mathrm{L}=$ detector distance (meters); $\mathrm{V}=$ vehicle speed $(\mathrm{km} / \mathrm{h}) ; \mathrm{UE}=$ vehicle interval (seconds); 5.5 $=$ average vehicle length (meters); $3=$ desired allowable gap (seconds); and $0.28=$ factor converting the vehicle speed from $\mathrm{km} / \mathrm{h}$ to meters/second.

To determine the appropriate location of the platoon detector, the distance between the conventional vehicle detector and the platoon detector $\Delta \mathrm{L}$, should be large enough to provide sufficient space for detecting a vehicle platoon. Based on similar concept as in Equation 1, $\Delta \mathrm{L}$ can be estimated in terms of platoon speed $\left(\mathrm{V}_{\mathrm{p}}\right)$, number of vehicle in the platoon $\left(\mathrm{N}_{\mathrm{p}}\right)$, and headway $\left(\mathrm{h}_{\mathrm{p}}\right)$ as shown in Equation 2.
(2) $\Delta \mathrm{L}=0.28 \mathrm{~V}_{\mathrm{p}}\left(\mathrm{N}_{\mathrm{p}}-1\right) \mathrm{h}_{\mathrm{p}}+5.5$

where the units for $\Delta \mathrm{L}, \mathrm{V}_{\mathrm{p}}$ and $\mathrm{h}_{\mathrm{p}}$ are meters, $\mathrm{km} / \mathrm{h}$, and seconds, respectively.

For highway corridors with high approach speed, adding a proper value of $\mathrm{L}$ to $\Delta \mathrm{L}$ gives the total setback of the platoon detector, $\mathrm{L}_{\mathrm{p}}$. For low-speed approaches, $L_{p}$ is the summation of Equation 1 and Equation 2, which can be simplified as:

(3) $\mathrm{L}_{\mathrm{p}}=0.28 \mathrm{~V}_{\mathrm{p}} \mathrm{N}_{\mathrm{p}} \mathrm{h}_{\mathrm{p}}$

where the units for $\mathrm{L}_{\mathrm{p}}, \mathrm{V}_{\mathrm{p}}$ and $\mathrm{h}_{\mathrm{p}}$ are meters, $\mathrm{km} / \mathrm{h}$, and seconds, respectively.

To use Equation 3, the values of $\mathrm{L}_{\mathrm{p}}, \mathrm{V}_{\mathrm{p}}$ and $\mathrm{h}_{\mathrm{p}}$ must be determined. However, the values of these variables are not constant and their representative values should be utilized to determine the detector location. With a given significance level, the platoon variables can be estimated with their statistical values, such as sample means and standard deviations. A random variable's mean, $\mu$, can be estimated using the sample mean $\overline{\mathrm{X}}$, the sample standard deviation $\mathrm{S}$, and the sample size $\mathrm{n}$ with a $100(1-\alpha) \%$ confidence interval (Neter, Wasserman, and Kutner 1985):

(4) $\bar{x} \quad t_{\alpha / 2, n 11} \frac{S}{\sqrt{n}} \leq \mu \leq \bar{x}+t_{\alpha / 2, n 1} \frac{S}{\sqrt{n}}$

where $t_{\alpha / 2, n-1}$ denotes the percentage point of the $\mathrm{t}$ distribution with $\mathrm{t}-1$ degrees of freedom. 
With Equation 3, the upper and lower bounds of $\mathrm{L}_{\mathrm{p}}$ can be estimated in terms of the sample mean values of $\mathrm{V}_{\mathrm{p}}, \mathrm{N}_{\mathrm{p}}$ and $\mathrm{h}_{\mathrm{p}}$. Set $\alpha=$ 0.05 and $\lambda=t_{a / 2, n-1}$, then $\lambda=1.96$ when sample size $n \geq 120$. Thus, the upper bound of the platoon detector distance $\mathrm{UL}_{\mathrm{p}}$ (in meters) can be estimated by substituting

$\mathrm{V}_{p}=\overline{\mathrm{V}}_{p}\left(1+\lambda \frac{\mathrm{S}_{\mathrm{V}_{\mathrm{p}}}}{\sqrt{\mathrm{n}}}\right), \quad \mathrm{N}_{\mathrm{p}}=\overline{\mathrm{N}}_{\mathrm{p}}\left(1+\lambda \frac{\mathrm{S}_{\mathrm{N}_{\mathrm{p}}}}{\sqrt{\mathrm{n}}}\right)$

and $h_{p}=\bar{h}_{p}\left(1+\lambda \frac{S_{h_{p}}}{\sqrt{n}}\right)$ into Equation 3:

(5) $\mathrm{UL}_{\mathrm{p}}=0.28 \overline{\mathrm{V}}_{\mathrm{p}} \overline{\mathrm{N}}_{\mathrm{p}} \overline{\mathrm{h}}_{\mathrm{p}}\left(1+\lambda \frac{\mathrm{S}_{\mathrm{V}_{\mathrm{p}}}}{\sqrt{\mathrm{n}}}\right)\left(1+\lambda \frac{\mathrm{S}_{\mathrm{N}_{\mathrm{p}}}}{\sqrt{\mathrm{n}}}\right)\left(1+\lambda \frac{\mathrm{S}_{\mathrm{h}_{\mathrm{p}}}}{\sqrt{\mathrm{n}}}\right)$ where $\overline{\mathrm{V}}_{\mathrm{p}}, \overline{\mathrm{N}}_{\mathrm{p}}$ and $\overline{\mathrm{h}}_{\mathrm{p}}$ are the mean values of the platoon speed $(\mathrm{km} / \mathrm{h})$, size (number of vehicles), and headway (seconds) from $\mathrm{n}$ measured platoons, respectively; and $\mathrm{S}_{\mathrm{vp}}$, $\mathrm{S}_{\mathrm{Np}}$ and $\mathrm{S}_{\mathrm{hp}}$. are their corresponding standard deviations.

Similarly, the lower bound of the platoon detector distance $\mathrm{LL}_{\mathrm{p}}$ can be estimated as:

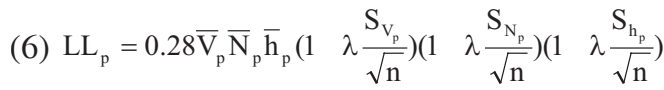

Using Equations 5 and 6, the platoon detector distances were calculated with collected traffic platoon data at eight isolated highway intersections. Each of the eight intersections consists of a major highway and a minor road (such as a county road with low traffic volume). The adjacent upstream intersection of each selected intersection is at least two miles (3.2 kilometers) away. Table 1 presents the $\mathrm{UL}_{\mathrm{p}}$ and $\mathrm{LL}_{\mathrm{p}}$ values at the intersections. In the table, each of the intersections is represented by the major highway at the intersection.

The pair of $\mathrm{LL}_{\mathrm{p}}$ and $\mathrm{UL}_{\mathrm{p}}$ values at each intersection provides a range of platoon detector distance values that can be utilized to provide sufficient platoon-based traffic information. That is, a platoon detector placed at a distance between the calculated $\mathrm{LL}_{\mathrm{p}}$ and $\mathrm{UL}_{\mathrm{p}}$ should be able to obtain statistically sufficient and stable platoon data. To verify this, platoon-based traffic data was collected at the intersection at US 31 with two vehicle counters. As shown in Table 1 , the $\mathrm{LL}_{\mathrm{p}}$ is 817 meters and $\mathrm{UL}_{\mathrm{p}}$ is 1,661 meters at the intersection. Thus, one traffic counter was placed at about 820 meters from the stop line and the other traffic counter was placed at about 1,600 meters. That is, both counters were within the range formed by $\mathrm{LL}_{\mathrm{p}}$ and $\mathrm{UL}_{\mathrm{p}}$ with one near the lower boundary and the other near the upper boundary. There are no side roads between these two locations. The measured data is summarized in Figures 5, 6 and 7. Figure 5 illustrates the traffic volumes measured during a 12-hour period at the two counter locations. It shows that the volume measurements at these two locations follow the same pattern. Similarly, Figures 6 and 7 show that at the two counters, the proportions of platooned vehicles and the maximum platoon sizes also exhibit the same trend. This implies that a platoon detector placed within the interval between $\mathrm{LL}_{\mathrm{p}}$ and $\mathrm{UL}_{\mathrm{p}}$ can provide consistent and stable platoon information.

The purpose of placing a platoon detector at an appropriate location is to capture the pertinent information of vehicle platoons. Based on the information, a platoon-based signal-timing method could be developed. With such a signal-timing method, the calculated platoon detector distance would leave sufficient time for the intersection signal controller device to adjust signal timing to accommodate the approaching vehicle platoons. In reality, the platoon configuration changes as a vehicle platoon travels through time and space. As shown in Figures 5 through 7, the changes of configuration are not significant over a distance of 840 meters. As shown in Table 1, the platoon detector distances range from about 400 meters to 1,600 meters. In terms of signal-timing variables, the minor changes in platoon configurations over a platoon detector distance will not significantly

Table 1: Estimated Platoon Detector Locations (meters)

\begin{tabular}{|c|c|c|c|c|c|c|c|c|}
\hline Intersection & US 52 & US 31 & SR 2 & SR 332 & SR 37 & SR 66 & US 27 & US 30 \\
\hline LL $_{\mathbf{p}}$ (meters) & 799 & 817 & 402 & 412 & 396 & 396 & 408 & 597 \\
\hline $\mathbf{U L}_{\mathbf{p}}$ (meters) & 1524 & 1661 & 869 & 665 & 857 & 890 & 835 & 1247 \\
\hline
\end{tabular}

US US Highways SR State Roads 
affect the resulting traffic-control strategy. Therefore, the changes of platoon configuration between the platoon detector and the stop line were not analyzed in this study.

\section{MATHEMATICAL DISTRIBUTIONS OF PLATOON VARIABLES}

As discussed above, the key platoon variables were selected, the critical platoon headway was chosen, and the method for determining platoon detector locations was developed. The selected platoon variables, along with the appropriate critical platoon headway and detector location, provide a basis for platoonbased traffic data collection and analysis. To study the characteristics of the platoon variables, isolated intersections were selected for platoon data measurements on Indiana highway corridors based on a statewide traffic survey. For the purpose of this study, each of the selected intersections includes a major road with relatively high traffic volume and a minor road with very low traffic volume. A large amount of platoon-based traffic data was collected at the intersections with traffic counters placed at calculated locations. Vehicle platoons were characterized using platoon size, platoon headway, platoon speed, and inter-arrival time between consecutive platoons as previously defined. Based on the frequency distributions of the four platoon key variables, three mathematical distributions were selected to fit the traffic data, including the negative exponential distribution, the normal distribution, and the lognormal distribution. Detailed descriptions of

Figure 5: Platoon Flow at Two Counter Locations

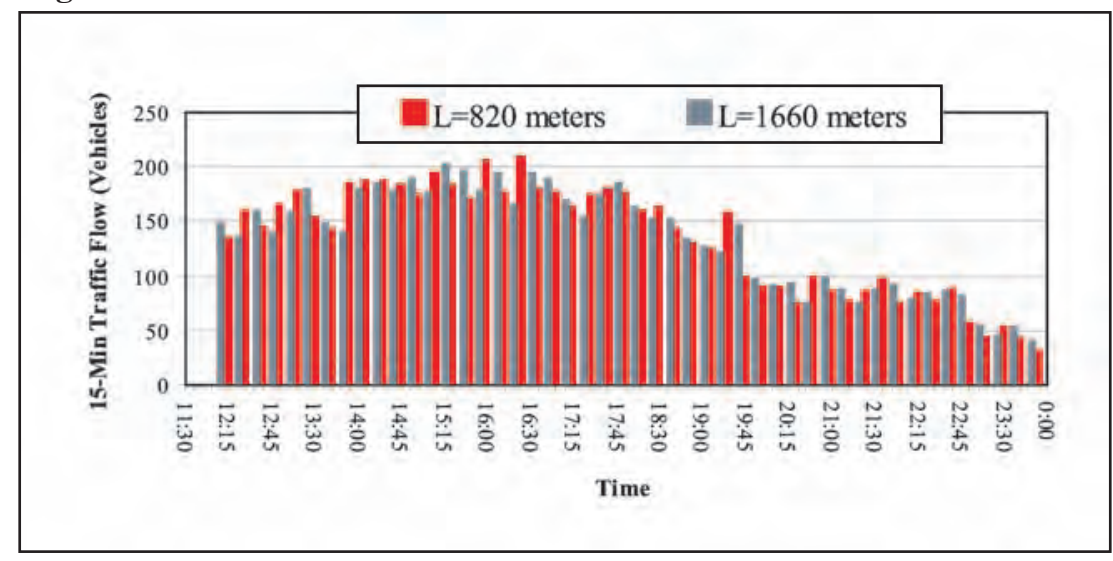

Figure 6: Percentage of Platooned Vehicles at Two Counter Locations

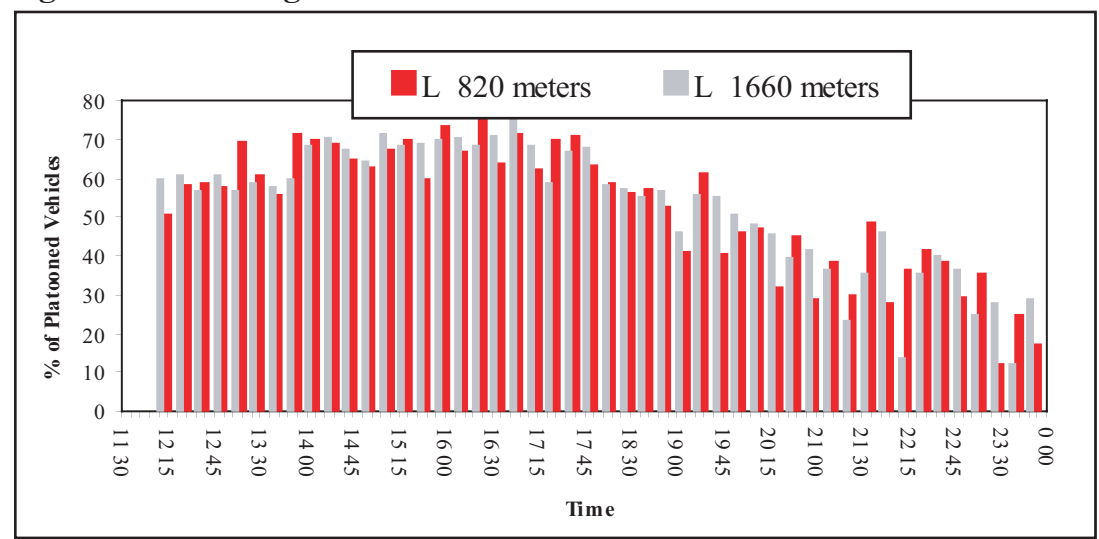


Figure 7: Maximum Platoon Sizes at Two Counter Locations

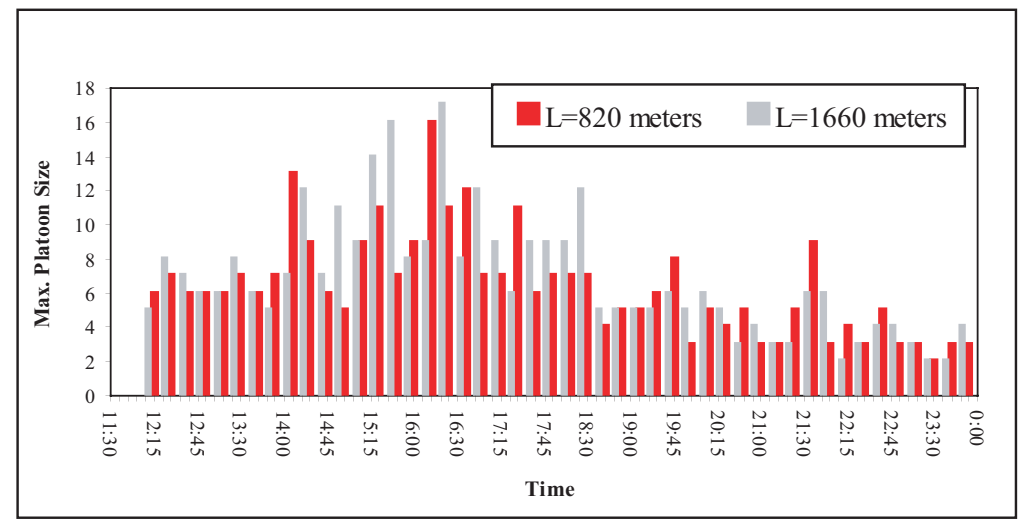

various mathematical distributions can be found in many books on probability and statistics, such as Walpole and Myers (1972) and Neter, Wasserman and Kutner (1985). An excellent reference on modeling traffic flows with the mathematical distribution models is Gerlough and Huber (1975).

The negative exponential distribution has the following form:

$$
\text { (7) } P(x \geq s) \quad e^{s / s}
$$

where $\mathrm{P}(\mathrm{x} \geq \mathrm{s})$ is the probability of a random variable $\mathrm{x}$ equal to or greater than a specified value $\mathrm{s}$, and $\mathrm{S}$ is the mean of the observed values of variable $\mathrm{x}$.

The normal distribution $\mathrm{N}(\mu, \sigma)$ is characterized by the mean $\mu$ and standard deviation $\sigma$ of a random variable $\mathrm{x}$. With the following conversion, the normal distribution $\mathrm{N}(\mu, \sigma)$ can be transformed into the standard normal distribution $\mathrm{N}(0,1)$, with mean 0 and standard deviation 1.

$$
Z_{i} \frac{x_{i} \mu}{\sigma}
$$

where $\mu$ and $\sigma^{2}$ can be estimated with observed $\mathrm{x}$ values:

(9) $\hat{\mu}=\bar{x}=\frac{\sum_{i=1}^{N} x_{i}}{N}$

(10) $\hat{\sigma}^{2} \quad S_{s}^{2} \frac{\sum_{i=1}^{N}\left(x_{i} \bar{x}\right)^{2}}{N 1}$
The lognormal distribution is used to describe systems where the logarithm of the measured variable is normally distributed. If the measured variable is $x_{i}$, then $s_{i}=\log x_{i}$ is assumed to be normally distributed with estimated mean $\hat{\mu}$ and variance $\bar{\sigma}^{2}$ :

$$
\hat{\mu} \quad \bar{s} \frac{\sum_{i 1}^{N} s_{i}}{N} \frac{\sum_{i=1}^{N} \log x_{i}}{N}
$$

(12) $\hat{\sigma}^{2}=S_{s}^{2}=\frac{\sum_{i 1}^{N}\left(s_{i}-\bar{s}\right)^{2}}{N-1}=\frac{\sum\left(\log x_{i}-\bar{s}\right)^{2}}{N-1}$

\section{Platoon Size Distribution}

When analyzing vehicle platoons, a vehicle is usually classified either as a platooned vehicle or as a non-platooned vehicle. This makes it inconvenient for platoon analysis because of the random positions of the platooned vehicles and non-platooned vehicles within a traffic stream. It is therefore desired to analyze platoon-based traffic flows without separating platooned vehicles from non-platooned vehicles. This can be achieved by treating non-platooned vehicles as single-vehicle platoons. That is, each non-platooned vehicle is considered a special vehicle platoon with platoon size equal to one. Consequently, no vehicles are excluded from the platoon-based traffic flows and vehicle platoon sizes range from one to any number of consecutive vehicles with headways less than the critical headway of 2.5 seconds. By introducing single-vehicle platoons, it significantly simplifies 
the procedures for analyzing and simulating platoon behaviors because a single mathematical distribution model can represent both platoon and non-platooned vehicles.

Figure 8 illustrates the observed platoon size distributions at two intersections during peak hours based on the critical headway of 2.5 seconds. Two distribution curves are shown for each intersection, one including and the other excluding single-vehicle platoons. The two curves for each intersection indicate that the platoon size distributions have a similar pattern either including or excluding single-vehicle platoons. This implies that treating non-platooned vehicles as single-vehicle platoons will not change the platoon variable's mathematical distribution. Figure 8 also exhibits that the frequency of single vehicle platoons (platoon size of 1) was about $30 \%$. In other words, approximately $70 \%$ of the vehicles traveled in groups (with platoon size of two or more). Based on the shape of the platoon size distribution curves in Figure 8, two possible mathematical distributions, the negative exponential and lognormal distributions, were selected to fit the measured platoon size data.

Traffic measurements at three intersections were utilized for fitting the mathematical distributions. The expected platoon size frequencies for each distribution model were calculated to compare with the observed frequencies. $\chi^{2}$ goodness-of-fit tests (Walpole and Myers 1972) were conducted to determine which of the distributions could best represent the actual platoon size distribution. The observed and expected platoon size distributions along with the goodness-of-fit test results are presented in Table 2.

At a significance level of $\alpha=0.05$, the critical value of the goodness-of-fit test is $\chi_{0.95}^{2}(9)=19.02$. A goodness-of-fit test between observed frequency $\mathrm{O}_{\mathrm{i}}$ and expected frequency $\mathrm{E}_{\mathrm{i}}$ is based on the quantity. $x^{2}=\sum_{i=1}^{k} \frac{\left(O_{i}-E_{1}\right)^{2}}{E_{i}}$

If $\chi^{2} \leq \chi_{0.95}^{2}(9)=19.02$, the fit is good; otherwise, the fit is poor. The test results show that the negative exponential distribution fits the platoon size distributions at the US 30 and US 52 intersections at $\alpha=0.05$. However, both distributions were rejected by the goodness-of-fit tests for the given $\alpha$ value for the platoon size distribution at the US 31 intersection. Under this situation that not all of the distribution models fit a common distribution, selection of an appropriate distribution involves some practical considerations and judgments. Generally, the selected distribution should represent the shape of the natural spread of the actual data measurements. In addition, a distribution should not be too sophisticated for practical applications. Therefore, based on the goodness-of-fit test results and the fact that the general shape of the distribution curve at US 31 follows the same trend as those at the other two locations, the negative exponential distribution was selected to represent platoon size distribution.

\section{Platoon Headway Distribution}

Platoon headway is defined as the average of individual vehicle headways within a vehicle platoon. This definition is a simplification of the actual headways within a platoon because the individual vehicle headways within a platoon are unequal in reality. Because this definition does not change the relative temporal and spatial positions of the vehicle platoons in the traffic stream, the simplification will not affect the characteristics of the platoon-based traffic flows. When analyzing headway distributions of individual vehicles, lognormal distributions were often utilized to fit headway data (Daou 1964 and May 1965). However, individual vehicle headways and platoon headways differ in statistical characteristics. Figure 9 shows the distributions of the individual and platoon headways measured at the US 31 intersection. As can be seen, the platoon headway distribution exhibits a symmetrical pattern while the individual vehicle headway distribution skews to the left and spreads over a wide range. The difference in distributions is attributed to the fact that the maximum platoon headway is limited to 2.5 seconds, but the individual vehicle headway has no upper limit. The symmetrical pattern suggests that the platoon headways more likely follow a normal distribution rather than a lognormal distribution.

Platoon headway frequencies at two intersections are plotted in Figure 10. The platoon headways at both intersections distribute symmetrically around the mode of approximately 1.5 seconds. The goodness-of-fit tests were 


\section{Figure 8: Platoon Size Distributions at Two Intersections}
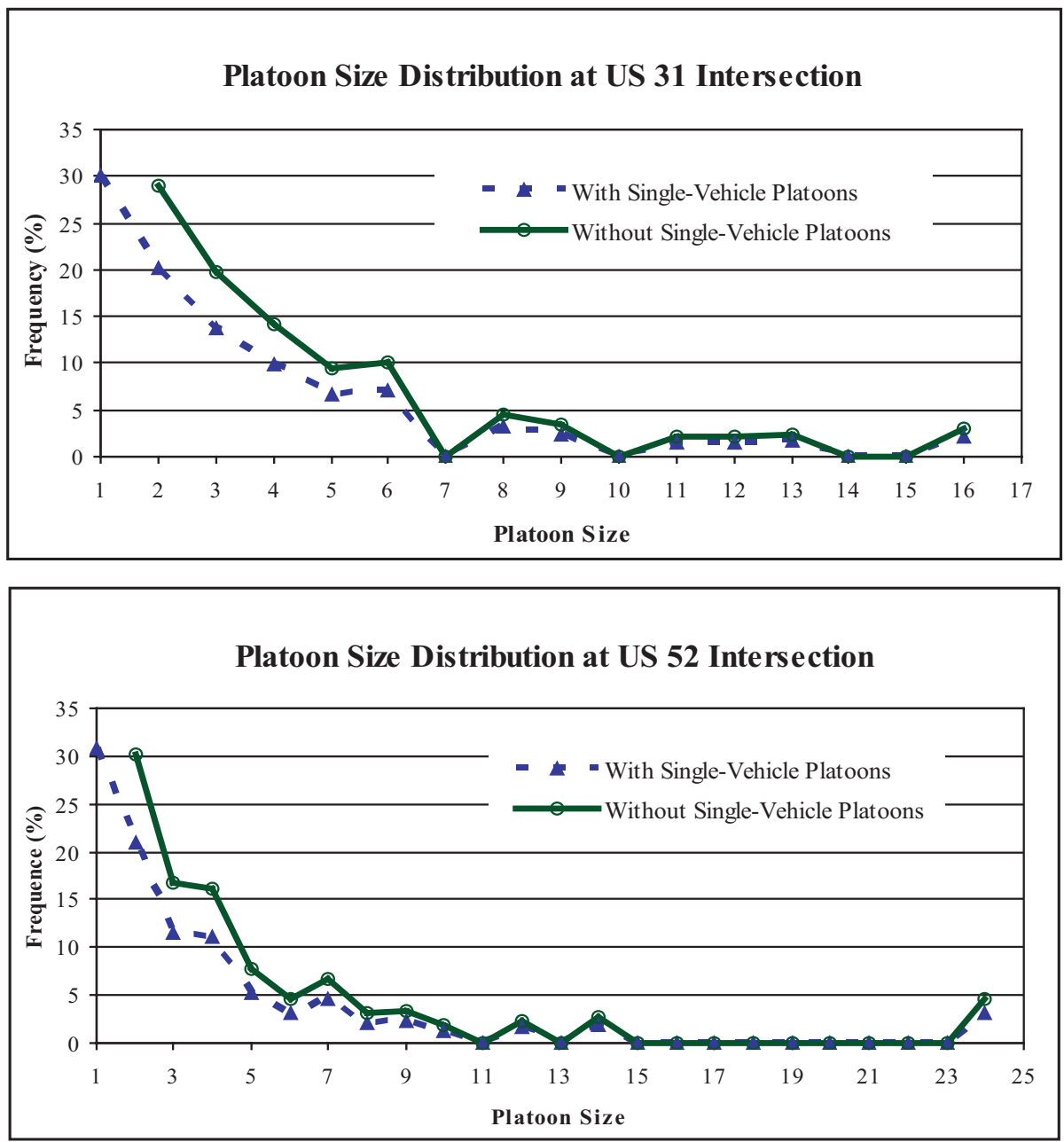

conducted to determine which distribution model, the normal distribution or the lognormal distribution, should be used for platoon headway distribution. As presented in Table 3, the platoon measurements were divided into six cells with a headway interval of 0.4 seconds for $\chi^{2}$ tests. At a significance level of 0.05 , the critical value is $\chi_{0.95}^{2}(5)=11.07$. For the US 52 intersection, the normal distribution is accepted and the lognormal distribution is rejected. However, for the US 31 intersection, both normal and lognormal distributions are accepted. This leads to the conclusion that the normal distribution model should be used to represent the platoon headway distributions. Moreover, the test results also indicate that in some cases, such as at the
US 31 intersection, the platoon headways could be represented by more than one mathematical distribution. Generally, when more than one mathematical distribution can be utilized, it is advisable to choose a model that can simplify the analysis procedure without sacrificing the accuracy. Since the normal distribution is simpler to use than the lognormal distribution, the normal distribution was selected.

\section{Platoon Inter-Arrival Time Distribution}

A platoon inter-arrival time is defined as the time interval between two successive platoons. That is, platoon inter-arrival time is the headway between the last vehicle of a vehicle platoon and 
Table 2: Goodness-of-Fit Tests for Platoon Size Distributions

\begin{tabular}{|c|c|c|c|c|}
\hline & \multicolumn{2}{|c|}{ Negative Exponential } & \multicolumn{2}{|c|}{ Lognormal } \\
\hline $\begin{array}{l}\text { Platoon } \\
\text { Size }\end{array}$ & $\begin{array}{c}\text { Observed } \\
\text { Frequencies }\end{array}$ & $\begin{array}{c}\text { Expected } \\
\text { Frequencies }\end{array}$ & $\begin{array}{c}\text { Observed } \\
\text { Frequencies }\end{array}$ & $\begin{array}{c}\text { Expected } \\
\text { Frequencies }\end{array}$ \\
\hline \multicolumn{5}{|c|}{ Intersection at US 30 one mile east of I 65 (Valparaiso) } \\
\hline 1 & 93 & 93.43 & 93 & 15.56 \\
\hline 2 & 54 & 68.74 & 54 & 25.40 \\
\hline 3 & 39 & 50.57 & 39 & 17.57 \\
\hline 4 & 27 & 37.20 & 27 & 10.95 \\
\hline 5 & 20 & 27.37 & 20 & 7.14 \\
\hline 6 & 17 & 20.14 & 17 & 4.57 \\
\hline 7 & 13 & 14.81 & 13 & 3.20 \\
\hline 8 & 10 & 10.90 & 10 & 2.18 \\
\hline 9 & 7 & 8.02 & 7 & 1.55 \\
\hline \multirow[t]{2}{*}{$>10$} & 3 & 5.90 & 3 & 4.89 \\
\hline & \multicolumn{2}{|c|}{$\chi^{2}=11.65<\chi_{0.95}^{2}=19.02$} & \multicolumn{2}{|c|}{$\chi^{2}=45.69>\chi_{n .95}^{2}=19.02$} \\
\hline \multicolumn{5}{|c|}{ Intersection at US 31 and Apperson Way (Kokomo) } \\
\hline 1 & 101 & 62.1 & 101 & 32.6 \\
\hline 2 & 49 & 38.2 & 49 & 37.0 \\
\hline 3 & 25 & 23.5 & 25 & 19.0 \\
\hline 4 & 13 & 14.5 & 13 & 9.0 \\
\hline 5 & 7 & 8.9 & 7 & 4.5 \\
\hline 6 & 5 & 6.5 & 5 & 2.3 \\
\hline 7 & 2 & 3.4 & 2 & 1.3 \\
\hline 8 & 2 & 2.1 & 2 & 0.7 \\
\hline 9 & 2 & 1,3 & 2 & 0.4 \\
\hline \multirow[t]{2}{*}{$>10$} & 1 & 0.8 & 1 & 0.8 \\
\hline & \multicolumn{2}{|c|}{$\chi^{2}=29.6>\chi_{0.95}^{2}=19.02$} & \multicolumn{2}{|c|}{$\chi^{2}=24.1>\chi_{0.95}^{2}=19.02$} \\
\hline \multicolumn{5}{|c|}{ Intersection at US 52 and Duncan Road (Lafayette) } \\
\hline 1 & 77 & 59.15 & 77 & 14.09 \\
\hline 2 & 42 & 45.44 & 42 & 22.87 \\
\hline 3 & 33 & 34.91 & 33 & 16.54 \\
\hline 4 & 29 & 26.81 & 29 & 11.13 \\
\hline 5 & 21 & 20.60 & 21 & 4.69 \\
\hline 6 & 16 & 15.82 & 16 & 8.01 \\
\hline 7 & 13 & 12.16 & 13 & 3.65 \\
\hline 8 & 12 & 9.34 & 12 & 2.69 \\
\hline 9 & 9 & 7.17 & 9 & 1.95 \\
\hline \multirow[t]{2}{*}{$>10$} & 8 & 5.518 & 8 & 7.37 \\
\hline & \multicolumn{2}{|c|}{$\chi^{2}=8.35<\chi_{0.95}^{2}=19.02$} & \multicolumn{2}{|c|}{$\chi^{2}=55.4>\chi_{0.95}^{2}=19.02$} \\
\hline
\end{tabular}

the first vehicle of the following vehicle platoon. Figure 11 illustrates the distributions of platoon inter-arrival times measured at three intersections. It is observed that all three distribution curves skew to the left with a similar trend. The platoon inter-arrival times range from 3 seconds to 40 seconds. The minimum observed platoon inter-arrival time is restricted by the critical headway of 2.5 seconds. Table 4 summarizes the goodness-of-fit test results for normal and lognormal distributions.

To examine the effect of time intervals on data distribution, $\chi^{2}$ tests were performed with respect to different time intervals. The distributions of the observed platoon inter-arrival times were arranged according to time intervals of 1,2 and 3 seconds, with 28, 14 and 10 test cells, respectively. Table 4 contains the critical 
Figure 9: Distributions of Individual Headways and Platoon Headways

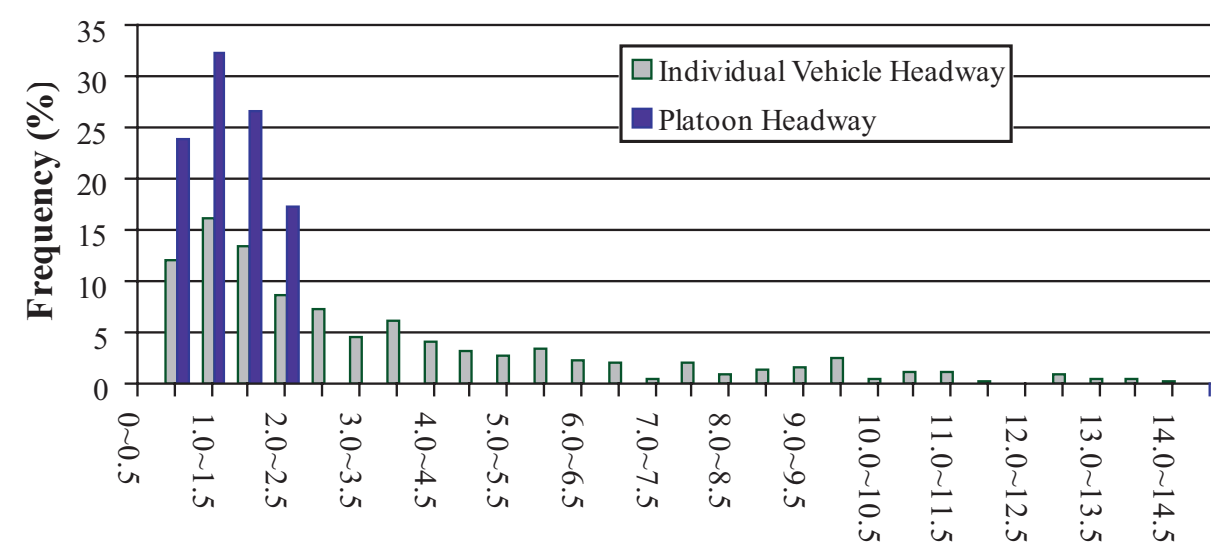

Headway Interval (Seconds)

\section{Figure 10: Measured Platoon Headway Distributions}

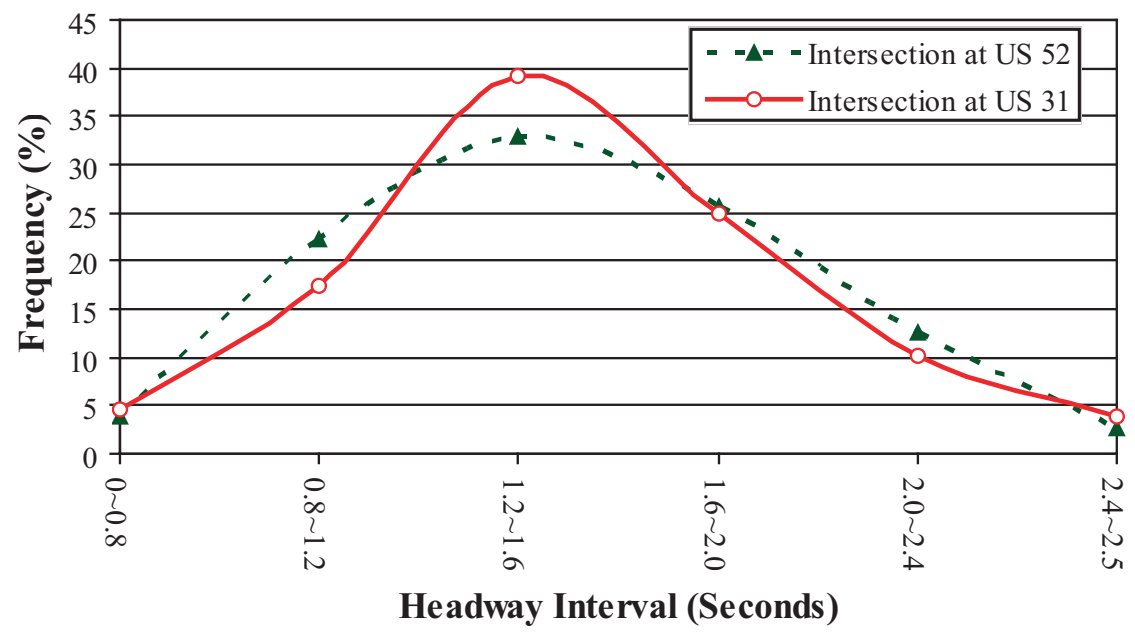

$\chi_{0.95}^{2}$ values and the computed $\chi^{2}$ values for the respective number of test cells. These values indicate that all the computed $\chi^{2}$ values exceed the corresponding critical $\chi_{0.95}^{2}$ for the normal distribution and all the computed $\chi^{2}$ values are less than the corresponding critical $\chi_{0.95}^{2}$ for the lognormal distribution. That is, with a significance level of 0.05 , the lognormal distribution model is accepted and the normal distribution model is rejected for the platoon inter-arrival time distribution.

\section{Platoon Speed Distribution}

Platoon speed is defined as the average speed of all vehicles in a platoon. Although the individual vehicle speeds within a platoon may not be exactly the same, the differences in vehicle speeds are expected to be minimal because of the constraint of the pre-determined critical headway. Plotted in Figure 12 are the distributions of the individual speed measurements and the platoon speed measurements at the US 31 intersection. 
Table 3: Goodness-of-Fit Tests for Platoon Headway Distributions

\begin{tabular}{|c|c|c|c|c|c|}
\hline \multicolumn{3}{|c|}{ Normal Distribution } & \multicolumn{3}{|c|}{ Lognormal Distribution } \\
\hline $\begin{array}{c}\text { Platoon } \\
\text { Headway (sec) }\end{array}$ & $\begin{array}{c}\text { Observed } \\
\text { Frequencies }\end{array}$ & $\begin{array}{c}\text { Expected } \\
\text { Frequencies }\end{array}$ & $\begin{array}{c}\text { Platoon } \\
\text { Headway (sec) }\end{array}$ & $\begin{array}{c}\text { Observed } \\
\text { Frequencies }\end{array}$ & $\begin{array}{c}\text { Expected } \\
\text { Frequencies }\end{array}$ \\
\hline \multicolumn{6}{|c|}{ Intersection at US 52 and Duncan Road (Lafayette) } \\
\hline$<0.80$ & 6 & 6.68 & $<0,80$ & 6 & 1.92 \\
\hline $0.80 \sim 1.20$ & 34 & 25.75 & $0.80 \sim 1.20$ & 34 & 31.39 \\
\hline $1.20-1.60$ & 50 & 50.78 & $1.20-1.60$ & 50 & 58.29 \\
\hline $1.60 \sim 2.00$ & 39 & 46.25 & $1.60 \sim 2.00$ & 39 & 38.92 \\
\hline $2.00-2.40$ & 19 & 19.45 & $2.00 \sim 2.40$ & 19 & 15.80 \\
\hline \multirow[t]{2}{*}{$>2.40$} & 4 & 4.10 & $>2.40$ & 4 & 6.68 \\
\hline & \multicolumn{2}{|c|}{$\chi^{2}=3.824<\chi_{0.95}^{2}=11.07$} & & \multicolumn{2}{|c|}{$\chi^{2}=16.56>\chi_{0.95}^{2}=11.07$} \\
\hline \multicolumn{6}{|c|}{ Intersection at US 31 and Apperson Way (Kokomo) } \\
\hline$<0.80$ & 7 & 9.5 & $<0.80$ & 7 & 3.8 \\
\hline $0.80 \sim 1.20$ & 27 & 31.2 & $0.80-1.20$ & 27 & 39.9 \\
\hline $1.20 \sim 1.60$ & 61 & 53.0 & $1.20-1.60$ & 61 & 58.6 \\
\hline $1.60 \sim 2.00$ & 39 & 42.9 & $1.60-2.00$ & 39 & 34.6 \\
\hline $2.00 \sim 2.40$ & 16 & 16.2 & $2.00-2.40$ & 16 & 13.4 \\
\hline \multirow[t]{2}{*}{$>2.40$} & 6 & 3.12 & $>2.40$ & 6 & 5.8 \\
\hline & \multicolumn{2}{|c|}{$\chi^{2}=5.46<\chi_{0.95}^{2}=11.07$} & & \multicolumn{2}{|c|}{$\chi^{2}=8.02<\chi^{2}{ }_{0.95}=11.07$} \\
\hline
\end{tabular}

The similarities of the two speed distributions are clearly illustrated by the two curves. Both the normal (Leong 1968) and lognormal (Haight and Mosher 1962) distribution models have been utilized for individual vehicle speeds. It appears in Figure 12 that the normal distribution may better represent the natural shape of the speed distribution.

Table 5 presents results of the $\chi^{2}$ goodnessof-fit tests conducted to fit the normal and lognormal distributions to the platoon speeds measured at the US 31 intersection. The critical $\chi_{0.95}^{2}$ value at a significance level of 0.05 is 11.07. Compared to the computed $\chi^{2}$ values, the normal distribution is accepted and the lognormal distribution is rejected. Therefore, the normal distribution model is recommended for the platoon speed distributions.

\section{VARIATIONS OF PLATOON VARIABLES}

Basic statistics of platoon variables, such as mean and standard deviation, are the basis of the platoon variable characteristics. As traffic flow rates change continuously, the platoon characteristics vary during different time periods. To reveal the statistics of platoon variables, the platoon traffic data was collected and analyzed with respect to four time durations. They include a 24-hour period, a 12-hour daytime period, a 4-hour period covering before, during, and after a peak hour, and a one peak-hour period. Since this study deals with only isolated intersections in rural or semi-urban areas, traffic flows are almost always non-congested at the selected intersections. The calculated statistics of platoon sizes, platoon headways, platoon speeds, and platoon inter-arrival times are presented in Figures 13, 14, 15, and 16, respectively. Note that the non-platooned vehicles are not included in the platoon size statistics. This is different from the platoon size distribution analysis that treated these vehicles as single-vehicle platoons to simplify the distribution model.

As demonstrated in Figure 13, the mean platoon size is three vehicles for the 24-hour and the 12-hour periods, and four vehicles for 4 -hour and 1-hour periods. The standard deviation also increases slightly as the time period shortens. This implies that as traffic volume increases, the mean platoon size decreases with slightly greater standard deviation. The fact that the mean platoon size increased by only one vehicle indicates that the majority of platooned vehicles travel in groups of three to four vehicles. 


Table 4: Goodness-of-Fit Tests for Platoon Inter-Arrival Times
\begin{tabular}{|c|c|c|c|c|c|}
\hline \multirow{3}{*}{ Intersection } & Interval & $\begin{array}{c}\text { Number of } \\
\text { Test Cells }\end{array}$ & $\chi_{0.95}^{2}$ & \multicolumn{2}{c|}{ Computed $\boldsymbol{\chi}^{2}$} \\
\cline { 4 - 6 } & I second & 28 & 40.11 & 115.82 & 24.52 \\
\cline { 3 - 6 } & 2 seconds & 14 & 22.36 & 86.17 & 11.74 \\
\cline { 2 - 6 } & 3 seconds & 10 & 16.92 & 74.24 & 9.27 \\
\hline \multirow{3}{*}{ US 52} & 1 second & 28 & 40.11 & 126.00 & 28.33 \\
\cline { 2 - 6 } & 2 seconds & 14 & 22.36 & 88.95 & 18.75 \\
\cline { 2 - 6 } & 3 seconds & 10 & 16.92 & 79.83 & 14.62 \\
\hline
\end{tabular}

Figure 11: Distributions of Inter-Arrival Time Measurements

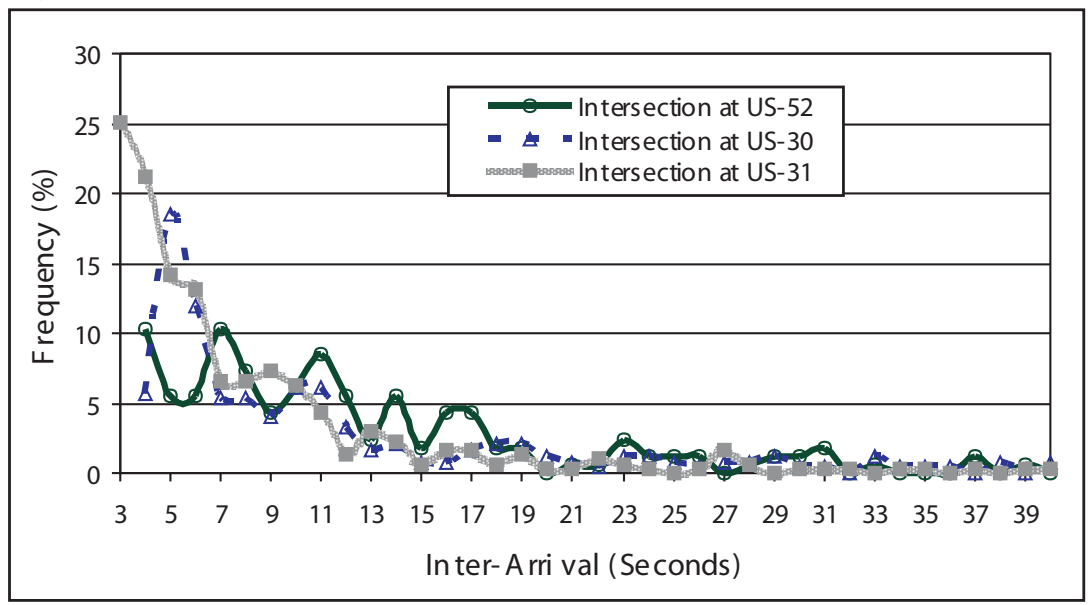

Figure 12: Individual Speed and Platoon Speed Distributions

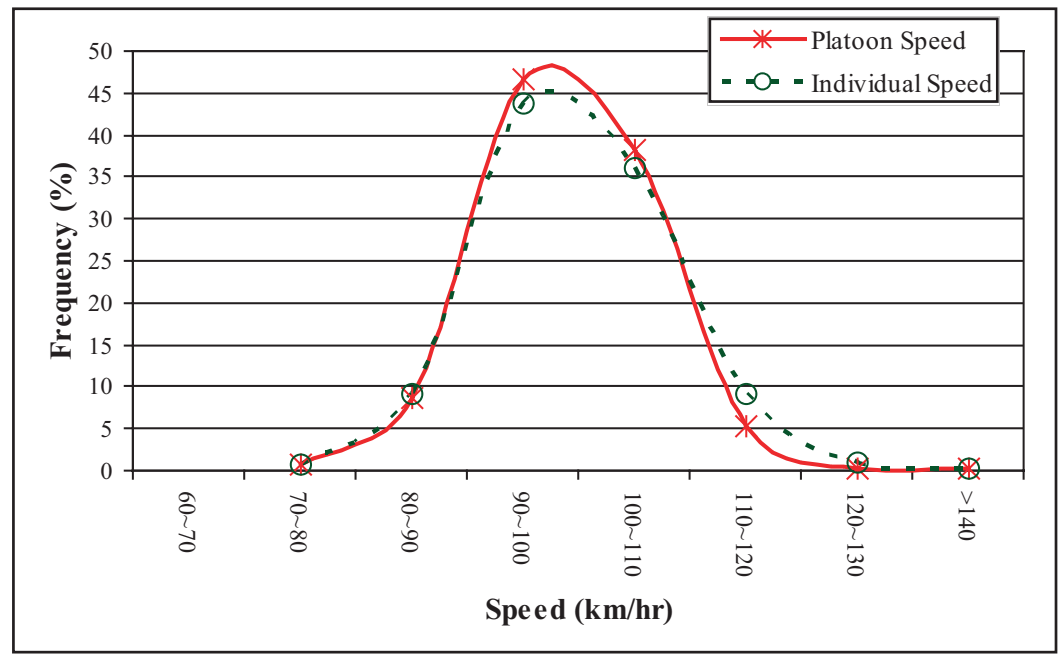




\section{Table 5: Goodness-of-Fit Tests for Platoon Speeds}

\begin{tabular}{|c|c|c|c|c|c|}
\hline \multicolumn{3}{|c|}{ Normal Distribution } & \multicolumn{3}{|c|}{ Lognormal Distribution } \\
\hline $\begin{array}{c}\text { Platoon Speed } \\
(\mathrm{km} / \mathrm{hr})\end{array}$ & $\begin{array}{c}\text { Observed } \\
\text { Frequencies }\end{array}$ & $\begin{array}{c}\text { Expected } \\
\text { Frequencies }\end{array}$ & $\begin{array}{l}\text { Platoon Speed } \\
(\mathrm{km} / \mathrm{hr})\end{array}$ & $\begin{array}{c}\text { Observed } \\
\text { Frequencies }\end{array}$ & $\begin{array}{c}\text { Expected } \\
\text { Frequencies }\end{array}$ \\
\hline \multicolumn{6}{|c|}{ Intersection at US 31 and Apperson Way (Kokomo) } \\
\hline $0 \sim 80$ & 3 & 1.23 & $0 \sim 80$ & 3 & 0.50 \\
\hline $80 \sim 90$ & 33 & 35.85 & $80 \sim 90$ & 33 & 34.66 \\
\hline $90 \sim 100$ & 179 & 173.58 & $90 \sim 100$ & 179 & 181.43 \\
\hline $100 \sim 110$ & 146 & 149.14 & $100 \sim 110$ & 146 & 141.56 \\
\hline $110 \sim 120$ & 20 & 22.67 & $110 \sim 120$ & 20 & 23.71 \\
\hline \multirow[t]{2}{*}{$>120$} & 2 & 0.54 & $>120$ & 2 & 1.15 \\
\hline & \multicolumn{2}{|c|}{$\chi^{2}=7.34<\chi_{0.95}^{2}=11.07$} & & \multicolumn{2}{|c|}{$\chi^{2}=14.03>\chi_{0.95}^{2}=11.07$} \\
\hline
\end{tabular}

\section{Different Time Periods}

Figure 14 clearly reveals the stability of platoon headways for different time periods. The means of platoon headways range from 1.47 to 1.50 seconds and the standard deviations are between 0.45 and 0.49 seconds for the four time periods. As defined earlier, a platoon headway is the average value of headways of all vehicles within a platoon. The statistics of platoon headways also indicate that the average perceived safe headway by motorists is about 1.5 seconds. The stability of platoon headway is attributed to the constraints of the perceived safe headway and the pre-determined critical headway value (2.5 seconds).

Figure 15 shows that the statistics of platoon speeds are also stable during the four time periods. This was expected because of the fact that the traffic flows were non-congested at the selected intersections. Figure 16 demonstrates that the values of mean and standard deviation of the inter-arrival time measurements decrease and stabilize as the time period shortens. This means that when traffic volume increases, the headways between vehicle platoons decreases and stabilizes at a certain level.

As the traffic flow rate increases, the average headway decreases. When the flow rates increase to a certain level, average headway will eventually fall below the specified critical headway of 2.5 seconds for identifying vehicle platoons. That is, all vehicles will be classified into a very long vehicle platoon at the saturated traffic flow rate. Therefore, the percentage of vehicles belonging to platoons can reach $100 \%$ as traffic flow rate increases. This percentage, named platoon fraction, is apparently a function of traffic flow rate. Figure 17 illustrates the relationship between the measured platoon fraction and the traffic flow rate. A regression equation between platoon fraction and traffic flow rate was obtained with the measured platoon-based traffic data. The coefficient of determination of the regression is $r^{2}=0.9213$.

$$
P_{\%}=1.6739 q^{0.70822}
$$

where $\mathrm{P}_{\%}=$ platoon fraction (\%), and $\mathrm{q}=15$ minutes flow rate (vehicles).

Similarly, the maximum platoon size is also affected by traffic flow rate and their relationship can also be established through regression. Figure 18 shows the relationship between the measured maximum platoon size and the traffic flow rate and Equation 14 is the corresponding regression equation (with $\mathrm{r}^{2}=0.905$ ).

$$
\mathrm{S}_{\max }=0.1313 \mathrm{q}^{0.7821}
$$

where $S_{\max }=$ maximum platoon size (vehicles); and $\mathrm{q}=15$-minute flow rate (vehicles).

As both the platoon fraction and maximum platoon size increase with traffic flow rate, an effort was also made to establish the relationship between the platoon fraction and maximum platoon size. It can be imaged that when the flow rate becomes saturated, the average headway will be below the selected critical headway so that the platoon fraction will approach $100 \%$ and 
Figure 13: Statistics of Platoon Sizes During Different Time Periods

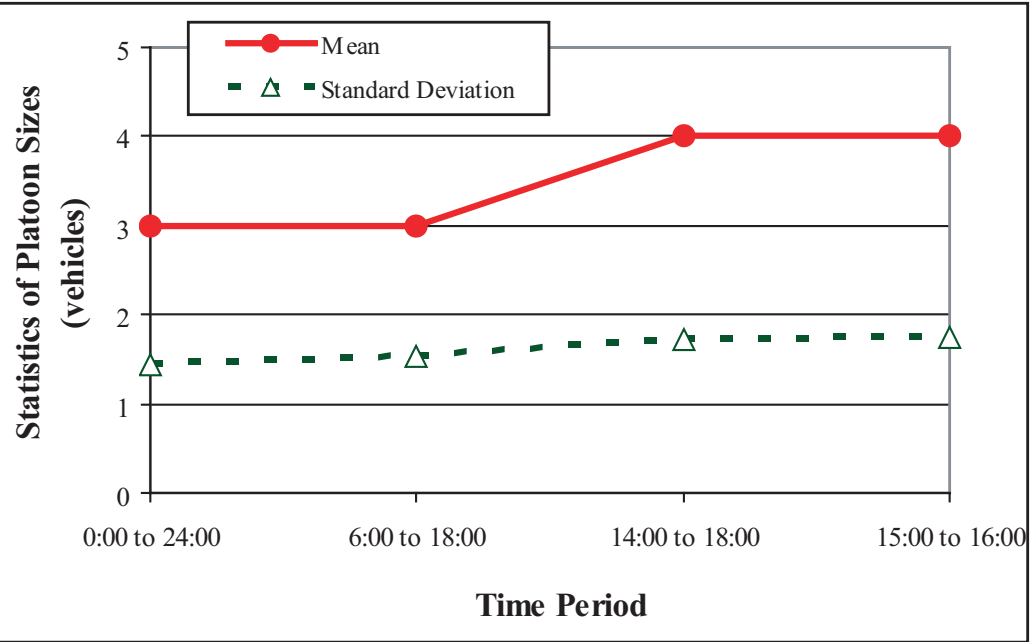

Figure 14: Statistics of Platoon Headways During Different Time Periods

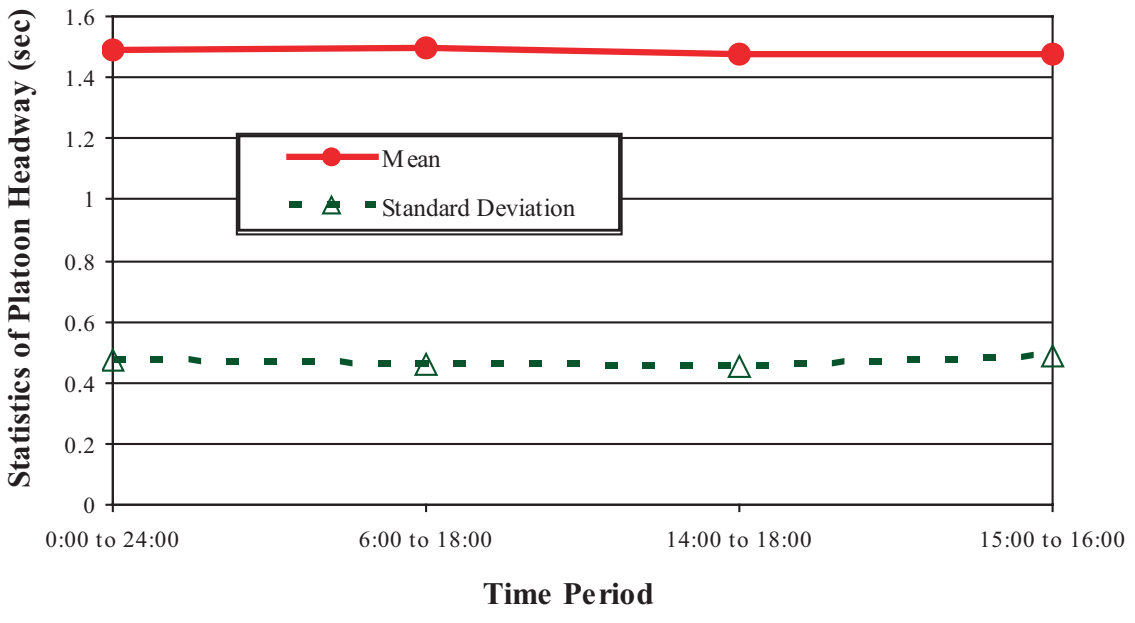


Figure 15: Statistics of Platoon Speeds During Different Time Periods

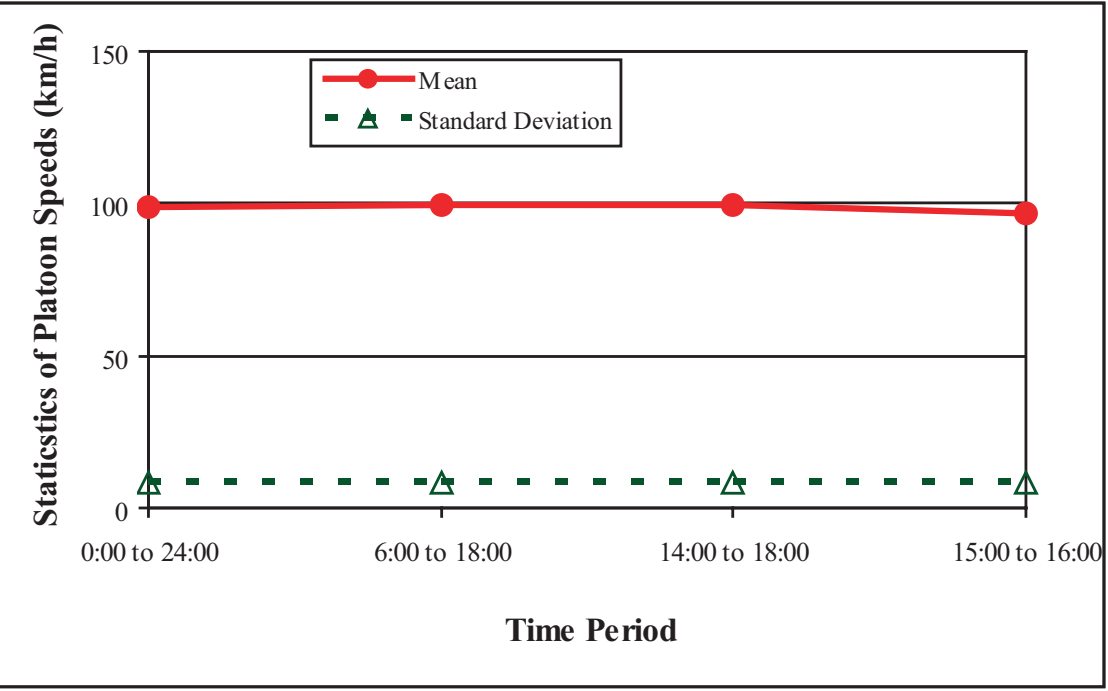

Figure 16: Statistics of Inter-Arrival Times During Different Time Periods

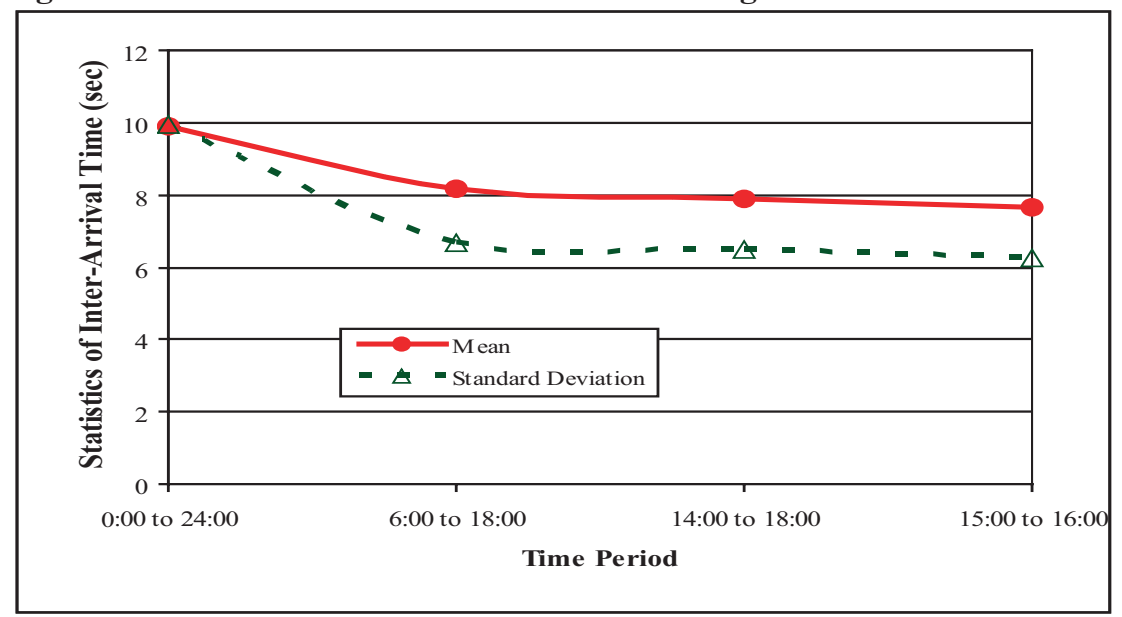


the maximum platoon size will include all the vehicles in the traffic stream. Figure 19 depicts the relationship between the measured maximum platoon size and the platoon fraction. The regression equation between the two quantities is given below (with $\mathrm{r}^{2}=0.846$ ):
(15) $\mathrm{S}_{\max }=0.1054 \mathrm{P}_{\%}^{0.9624}$

where $\mathrm{S}_{\max }=$ maximum platoon size, and $\mathrm{P}_{\%}=$ platoon fraction $(\%)$.

Figure 17: Platoon Fraction and Traffic Flow Rate Relationship

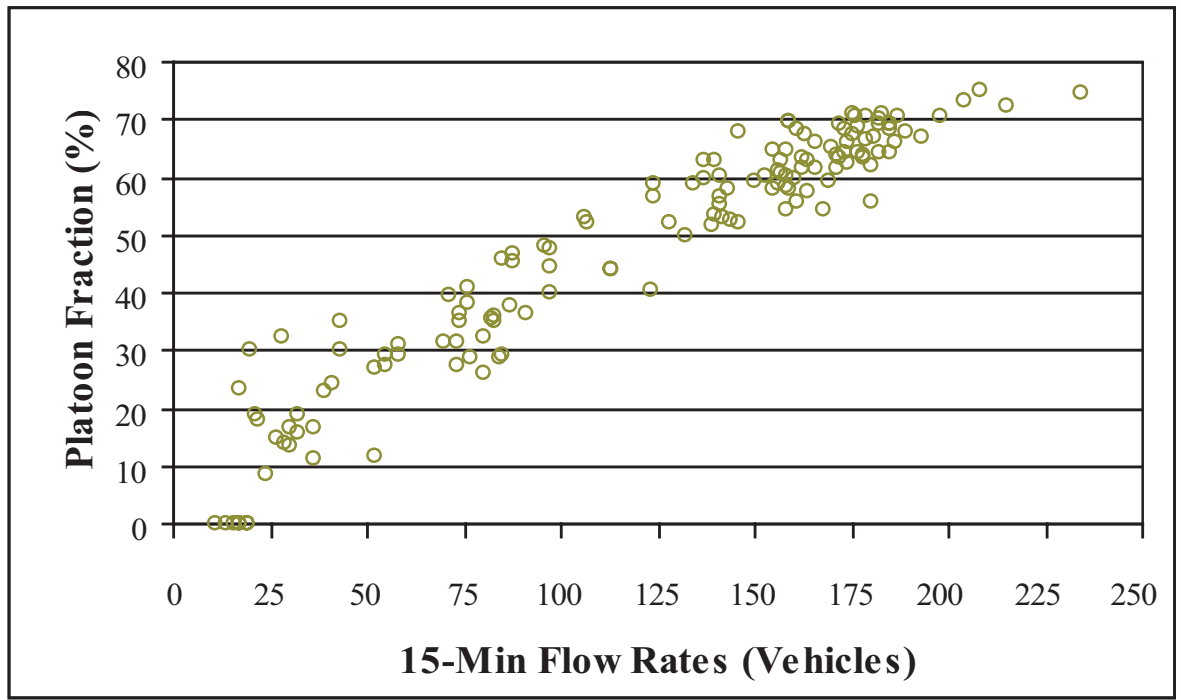

Figure 18: Maximum Platoon Size and Traffic Flow Rate Relationship

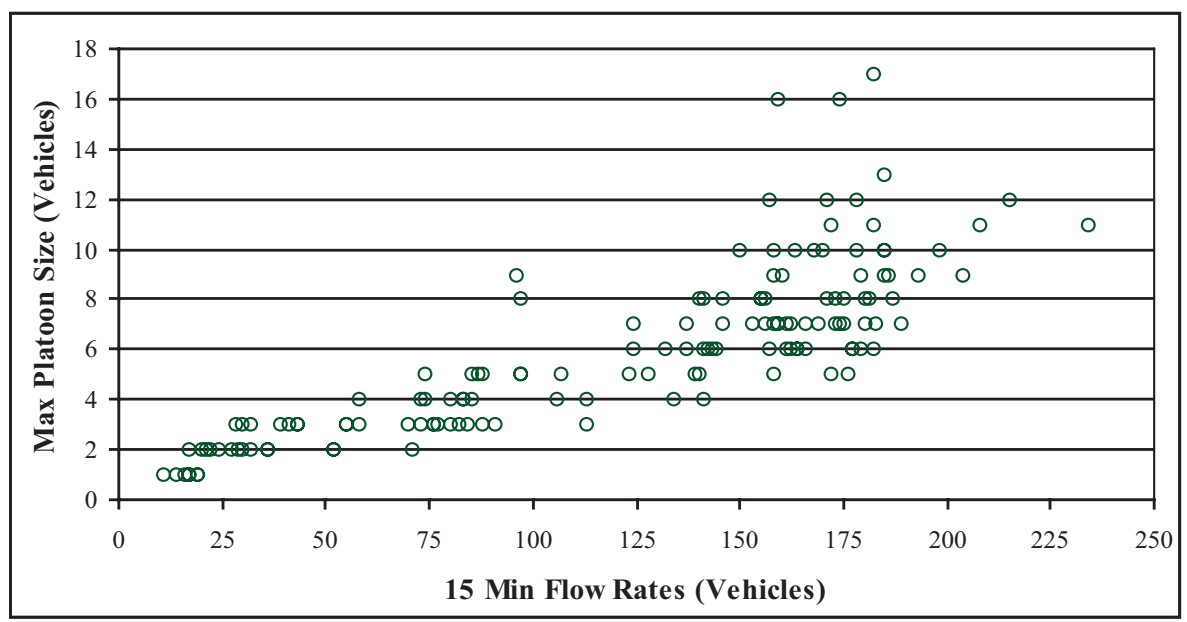


Figure 19: Maximum Platoon Size and Platoon Fraction Relationship

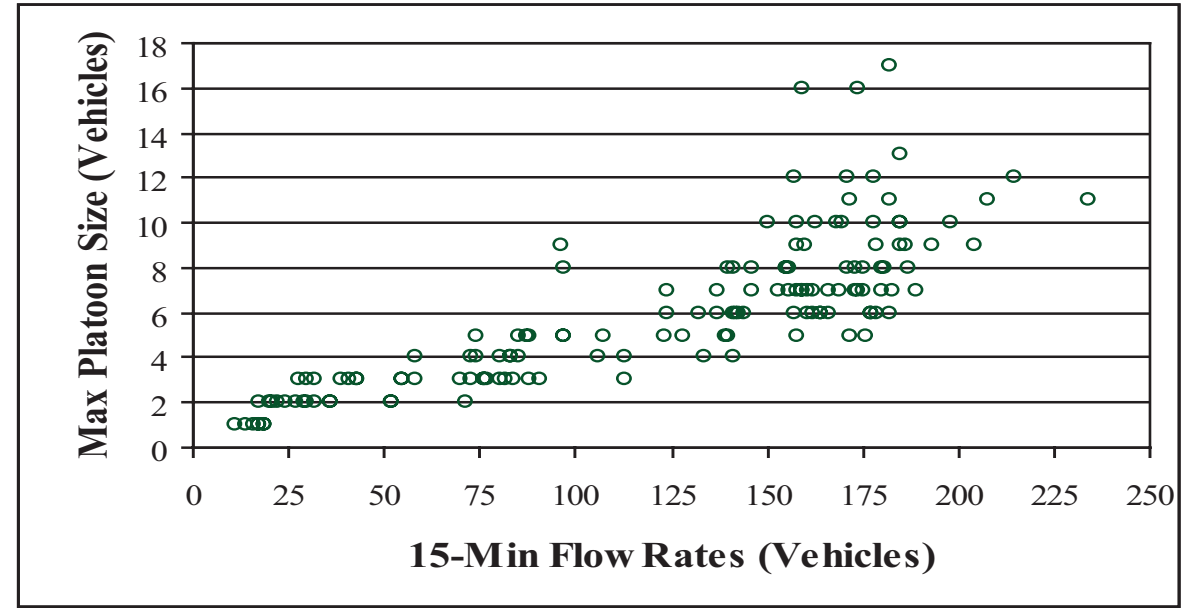

\section{CONCLUSIONS}

To effectively analyze traffic flows in terms of vehicle platoons, the following vital variables were selected and measured - the platoon size, the platoon headway, the platoon speed, and the platoon inter-arrival time. These four fundamental variables of vehicle platoons were analyzed to capture the characteristics of vehicle platoon movements. In addition, the critical headway of 2.5 seconds was determined to distinguish platooned vehicles from non-platooned vehicles. Equations were derived to determine the appropriate locations of platoon detectors. The distance of a platoon detector from the stop line at an intersection can be computed using the typical values of platoon speed, size, and headway. The equations assure that the produced platoon detector location would enable the detectors to obtain sufficient and stable platoon information. Through statistical analyses, the appropriate mathematical distributions of the vehicle platoon variables were determined. It was found that the platoon size follows the negative exponential distribution, the platoon headway has the normal distribution, the platoon inter-arrival time fits the lognormal distribution, and the platoon speed suits the normal distribution. The statistics of the key platoon variables at different traffic flow levels were computed based on the recorded traffic flow data. Also developed are the regression equations between various platoon related quantities, such as platoon fraction versus traffic flow rate, maximum platoon size versus traffic flow rate, and platoon fraction versus maximum platoon size. 


\section{REFERENCES}

Athol, P. "Headway Groupings.” Highway Research Record 72, Highway Research Board, National Research Council, Washington, D.C., 1965

Chaudhary, N. "PIA System Improves Traffic Flow and Reduces Wait Time." Texas Transportation Researcher, Volume: 39 Issue: 4, Texas Transportation Institute, Texas A\&M University, College Station, TX, 2003.

Daou, A. "The Distribution of Headways in a Platoon.” Operation Research 12(2), (1964).

Garber, N.J. and L.A. Hoel. Traffic and Highway Engineering, $3^{\text {rd }}$ Edition, PWS Publishing, Pacific Grove, California, 2002.

Gaur, A. and P. Mirchandani. "Method for Real-Time Recognition of Vehicle Platoons." Transportation Research Record 1748, Transportation Research Board, National Research Council, Washington, D. C., 2002.

Gerlough, D.L. and M.J. Huber. Traffic Flow Theory - A Monograph. Special Report 165, Transportation Research Board, National Research Council, Washington, D.C., 1975.

Haight, F.A. and W.W. Mosher. "A Practical Method for Improving the Accuracy of Vehicular Speed Distribution Measurements." Highway Research Record 341, Highway Research Board, National Research Council, Washington, D.C., 1962.

Institute of Transportation Engineers (ITE) "Small Area Detection at Intersection Approaches." Southern Section Technical Committee 18. Traffic Engineering 44(5), 1974.

Institute of Transportation Engineers (ITE) "Large-Area Detection at Intersection Approaches." Southern Section Technical Committee 17. Traffic Engineering 46(6), 1976.

Leong, H.J.W. "The Distribution and Trend of Free Speeds on Two-Lane Rural Highways in New South Wales.” Proceedings, Australian Road Research Board, Vol. 4, 1968.

May, A.D. "Gap Availability Studies." Highway Research Record 72, Highway Research Board, National Research Council, Washington, D.C., 1965.

May, A.D. Traffic Flow Fundamentals. Prentice Hall, Englewood Cliffs, New Jersey, 1990.

McShane, W.R., R.P. Roess, and E.S. Prassas. Traffic Engineering, $2^{\text {nd }}$ Edition, Prentice Hall, Upper Saddle River, New Jersey, 1998.

Neter, J., W. Wasserman, and M.H. Kutner. Applied Linear Statistical Models, $2^{\text {nd }}$ Edition, Richard D. Irwin, Inc., Homewood, Illinois, 1985.

Transportation Research Board (TRB). Highway Capacity Manual, $3^{\text {rd }}$ Edition, Special Report 209, National Research Council, Washington, D. C., 2000.

Walpole, R.E. and R.H. Myers. Probability and Statistics for Engineers and Scientists. The Macmillan Company, New York, 1972. 
Yi Jiang, Ph.D., P.E., is an assistant professor in the Department of Building Construction Management at Purdue University. His research interests include highway infrastructure management systems, highway construction, pavement performance evaluation and modeling, and traffic engineering. He received his M.S. and Ph.D. degrees in civil engineering from Purdue University and his B.S. degree in civil engineering from Tongji University in China.

Shuo Li, Ph.D., P.E., is a transportation research engineer with the Research Division, Indiana Department of Transportation. His research interests include fundamental data for highway infrastructure design, evaluation, and management, and smart technologies for pavement finite element method simulation, materials, and testing. 